\title{
Los órganos internacionales como herramienta en la lucha contra la pobreza en España después de la Gran Recesión (2008-2014)
}

\author{
International bodies as a tool in the fight against poverty \\ inspain after the Great Recession (2008-2014)
}

\author{
Silvia Avellaneda San Antonio \\ Universidad de Castilla-La Mancha \\ Silvia.avellaneda@uclm.es
}

Cómo citar/Citation: Avellaneda, Silvia. 2020. «Los órganos internacionales como herramienta en la lucha contra la pobreza en España después de la Gran Recesión (2008-2014)». Deusto Journal of Human Rights, No. 6: 125-151. doi: http://dx.doi.org/10.18543/djhr.1873.

Sumario: Introducción. 1. Tratados, informes y pronunciamientos. 1.1. Pobreza y derechos humanos. 1.2. Herramientas de protección internacional del derecho a un nivel de vida adecuado. El sistema de informes y sus pronunciamientos. 1.3. Los informes periódicos de España. 2. Recomendaciones y conclusiones de los órganos internacionales a los últimos informes de España. 2.1. Recomendaciones del Consejo de Derechos Humanos de Naciones Unidas. EPU de 2015. 2.2. Recomendaciones del CDESC de Naciones Unidas. Informe al PIDESC de 2017. 2.3. Conclusiones del CEDS del Consejo de Europa. Informes . $^{\circ} 29$ de 2016 y 31 de 2018. Conclusiones. Bibliografía.

Resumen: Este trabajo analiza las conclusiones y recomendaciones de órganos de Naciones Unidas y del Consejo de Europa, derivadas de los últimos informes periódicos de España, sobre la situación de los derechos directamente conectados con la pobreza: el derecho a un nivel de vida adecuado y a la protección económica y social. La conclusión es que estos órganos han mostrado su preocupación por la persistencia de altos niveles de pobreza en los años de recuperación económica y se han pronunciado sobre asuntos como la vivienda o las prestaciones; en términos de Derecho y de Derechos Humanos. El objetivo es contribuir al enfoque de los derechos humanos de la pobreza en España, argumentando que la lucha contra ella y la mejora de cuestiones concretas como la protección económica, la vivienda pública o la redistribución no son solo asuntos de agenda política sino también de cumplimiento de obligaciones internacionales contraídas por nuestro país. 
Palabras clave: derechos económico-sociales y culturales, derecho a un nivel de vida adecuado, comités internacionales, informes periódicos, pobreza.

Abstract: This paper analyses the conclusions and recommendations offered by bodies of the United Nations and the Council of Europe, derived from Spain's latest periodic reports, on the situation of rights directly connected to poverty, such as the right to an adequate standard of living and to economic and social protection. The conclusion is that these bodies have shown their concern about the persistence of high levels of poverty in the years of economic recovery and have expressed their views on matters such as housing and social benefits, in terms of both Law and Human Rights. The aim of the article is to contribute to the human rights approach to poverty in Spain, arguing that the fight against it and the improvement of specific issues such as economic protection, public housing or redistribution, are not only matters on the political agenda, but should also comply with international obligations assumed by our country.

Keywords: economic, social and cultural rights, right to an adequate standard of living, international committees, regular reports, poverty. 


\section{Introducción}

Entre enero y febrero de 2020 España recibió la visita del Relator Especial de Naciones Unidas sobre la Pobreza Extrema y los Derechos Humanos, Philip Alston, y en su comunicado anunciando esta visita expresó:

España es la quinta economía más grande de Europa y, sin embargo, tiene unos niveles de pobreza sorprendentemente altos. Esto plantea verdaderas preguntas sobre quién se ha beneficiado del reciente crecimiento y quién se ha quedado atrás (ONU 2020a).

En 2019, según el VIII Informe Foessa, en España había 8,5 millones de personas en situación de exclusión (el 18,4\% de la población), lo que suponía 1,2 de millones más que en el año 2007 (antes de la crisis de 2008). En términos generales, amplios sectores de población (el 48,4\%), integrados y que no tenían problemas de supervivencia, volvieron a los niveles anteriores a la Gran Recesión. Pero este informe pone de manifiesto la persistencia de amplias bolsas de pobreza y de sectores especialmente vulnerables donde efectivamente, "se ceba la desigualdad y la precariedad en sus diferentes formas: vivienda insegura e inadecuada, desempleo persistente, precariedad laboral extrema e invisibilidad para los partidos políticos» (FOESSA 2019). La recuperación económica a partir de 2014 llega con más dificultad a la población excluida: su tasa de exclusión de la vivienda ha aumentado un $2 \%$, mientras que la de la población general se reduce (Zugasti y García 2019, 6-7). En 2018, el 8,1\% de los hogares se encontraba en situaciones de exclusión severa, cifra muy similar a la de 2013, que se situaba en el 8,7\%. Ello implica que no se ha conseguido dar una respuesta a la situación de aquellos hogares y personas que peor estaban (Zugasti y García 2019, 25).

Y los últimos acontecimientos en España, en Europa y en el mundo no harán sino empeorar este panorama: la pandemia del COVID-19 tendrá consecuencias económicas y sociales devastadoras y no es aventurado asegurar que, de nuevo, los más golpeados serán los sectores y las familias más vulnerables para los que llueve sobre mojado. Tan solo durante la primera semana de confinamiento, Save the Children (2020) estima que el $60,5 \%$ de las familias a las que ya atendían antes de la emergencia ha visto alterada su situación laboral y el $41 \%$ está registrando estrés y problemas de convivencia. El caso más generalizado es aquél en el que ambos progenitores han perdido el empleo, en muchos casos ligado a la economía sumergida y 
excluidos de la protección social; o en el mejor de los casos, el trabajo se mantiene sin adecuada protección o el teletrabajo es anecdótico. Especialmente acuciante es la situación de los niños y niñas, sin colegio ni comedor, que en muchos casos $(48,6 \%)$ tienen dificultades para acceder a los materiales de los centros escolares.

La pobreza no es solo la falta de ingresos y recursos para garantizar unos medios de vida sostenibles, es un problema de derechos humanos; la pobreza erosiona o anula derechos económicos y sociales como el derecho a la salud, a una vivienda adecuada, a la alimentación y al agua potable, y el derecho a la educación; pero también derechos civiles y políticos, como el derecho a un juicio justo, la participación política o la seguridad de la persona (ACNUDH 2019). La lucha contra la pobreza es uno de los pilares fundamentales de los Objetivos de Desarrollo Sostenible (ODS) y la Agenda 2030 ofrece oportunidades decisivas para fomentar la aplicación de los derechos humanos. El Objetivo 1 es poner fin a la pobreza en todas sus formas; pero también es imperativo para todos los países, el hambre cero (Objetivo 2), garantizar la salud (Objetivo 3), la educación (Objetivo 4), el agua y el saneamiento (Objetivo 6) y el trabajo decente (Objetivo 8).

España forma parte del Sistema Internacional de Derechos Humanos y está obligada a cumplir los ODS y también a respetar, promover, proteger y hacer efectivos los derechos humanos, los derechos económicos, sociales y culturales (DESC) y el derecho a un nivel de vida adecuado. Debe rendir cuentas sobre el cumplimiento y respeto de los tratados internacionales y la situación de los derechos humanos, y para ello, entre otros mecanismos, debe presentar informes periódicos, a raíz de los cuales, los órganos y comités internacionales emiten unas conclusiones, observaciones y recomendaciones. En estos pronunciamientos, estos órganos delimitan un marco de referencia para los Estados de interpretación de los tratados y, sobre todo, determinan cumplimientos e incumplimientos $y$, en muchos casos, acciones y mejoras concretas que deben acometer para el efectivo respeto de los tratados de derechos humanos.

Con estas premisas, el objetivo general de este trabajo es analizar este marco de referencia de los derechos humanos directamente relacionados con la pobreza en España. Para ello, se plantean tres objetivos específicos: el primero hace referencia a qué debe cumplir nuestro país; el segundo a cómo debe rendir cuentas de ello. Para responder a estas cuestiones, en la primera parte introductoria (tratados, informes y pronunciamientos) se concretan los referentes internacionales y artículos relativos a los dos derechos directamente 
relacionados con la pobreza, el derecho a un nivel de vida adecuado y a la protección económica y social; se introducen las obligaciones de España con relación a la presentación de informes periódicos y se detallan los últimos ciclos de evaluación desde 2014.

El tercer objetivo específico, y principal, es concretar cuáles son las cuestiones más relevantes y las obligaciones específicas que los órganos internacionales han determinado que España debe acometer para el efectivo cumplimiento de estos derechos. Para ello le metodología usada ha sido el análisis de las recomendaciones y conclusiones del Consejo de Derechos Humanos y del Comité de Derechos Económicos, Sociales y Culturales (CDESC) de Naciones Unidas y del Comité Europeo de Derechos Sociales (CEDS) del Consejo de Europa, a raíz de los últimos cuatro informes de España. De esta forma, las dos principales aportaciones de este trabajo son: 1) un análisis trasversal y asociado de la apreciación de dos principales órganos de DESC y del Consejo de Derechos Humanos y, 2) la concreción de medidas ineludibles que España debe acometer para mitigar la pobreza, en términos de Derecho, obligaciones y tratados de derechos humanos.

\section{Tratados, informes y pronunciamientos}

\subsection{Pobreza y derechos humanos}

La Declaración Universal de Derechos Humanos (DUDH) de 1948 en su art. 25.1 enuncia:

Toda persona tiene derecho a un nivel de vida adecuado que le asegure, así como a su familia, la salud y el bienestar, y en especial la alimentación, el vestido, la vivienda, la asistencia médica y los servicios sociales necesarios; tiene asimismo derecho a los seguros en caso de desempleo, enfermedad, invalidez, viudez, vejez u otros casos de pérdida de sus medios de subsistencia por circunstancias independientes de su voluntad (ONU 1948).

Este derecho se desarrolla específicamente como DESC, en el art. 11.1 del Pacto Internacional de Derechos Económicos, Sociales y Culturales (PIDESC) de 1966; ratificado por España en 1977:

Los Estados Partes en el presente Pacto reconocen el derecho de toda persona a un nivel de vida adecuado para sí y su familia, incluso alimentación, vestido y vivienda adecuados, y a una mejora continua de las condiciones de existencia (ONU 1966). 
Ambas son las referencias fundamentales del derecho a un nivel de vida adecuado y concretan, asimismo, los derechos que lo integran: la alimentación adecuada, la vivienda, el agua y el saneamiento (Añón 2014), así como la protección social. En este sentido, el art. 9 del PIDESC, consagra el derecho a la seguridad social y a la asistencia social (ONU 1966). La definición de estos derechos, alcance e interpretación ha sido ampliamente desarrollada en las observaciones generales del CDESC, tales como su Observación General 4 (1991) y 7 (1997) sobre vivienda; Observación 12 (1999) sobre alimentación y 15 (2002) sobre agua; y sobre el derecho a la protección social (Observación General 19 aprobada en 2007) (CDESC 2020a).

En 1980 España ratifica el documento más importante sobre derechos sociales en Europa, la Carta Social Europa (CSE) de 1961 (Consejo de Europa 1961). Este texto no incorpora explícitamente el derecho a un nivel de vida adecuado; pero es el texto de referencia de la Europa social (Jimena Quesada 2014 y 2017). En esta línea, el Consejo de Europa y su CEDS han venido desarrollando una intensa labor en la lucha contra la pobreza haciendo una interpretación extensiva de artículos que no versan directamente sobre ello (Sanz 2012), y está siendo clave en cuestiones como la pobreza infantil, a través de la determinación de un conjunto clave de normas mínimas que salvaguardan los derechos de las familias y los niños, especialmente en tiempos de austeridad (Nolan 2019).

La CSE original de 1961 contempla contenidos y derechos relacionados con el derecho a un nivel de vida adecuado, tales como el derecho al trabajo, salarios justos, condiciones laborales, protección a la salud, a la familia o la niñez. De todos ellos, el art. 13 sobre el derecho a la asistencia social y médica establece, entre otros, que los Estados se comprometen a que:

Todas las personas puedan obtener por medio de servicios adecuados, públicos o privados, el asesoramiento y ayuda personal necesarios para prevenir, eliminar o aliviar su estado de necesidad personal o familiar (Consejo de Europa 1961).

Esta Carta se desarrolla en una Europa con los más altos niveles de desarrollo económico y del Estado del Bienestar, aun con las profundas diferencias entre los Estados parte. En este contexto, los sistemas de garantía y protección económica y social son elementos clave y precisamente el CEDS, con su interpretación extensiva, ha convertido la CSE en una herramienta de lucha contra la pobreza. Cobran así relevancia el derecho a la seguridad y a la asistencia social (art. 12 
al 14), el derecho de la familia (art. 16) y de las madres y los niños (art. 17) a una protección social, jurídica y económica.

En 1996 este texto se actualiza en la CSER que incluye importantes avances en materia de derechos sociales e igualdad y, además, reconoce, ahora sí, el derecho a la protección contra la pobreza y la exclusión social (art. 30) y a la vivienda (art. 31) (Consejo de Europa 1996). La inclusión de estos dos artículos supone dotar de entidad y protección jurídica a la lucha contra la pobreza (Terrádez 2019, 387) y, en sintonía con Naciones Unidas, incorporar un enfoque integral, complejo y universal. A comienzo de 2020, España no ha ratificado esta versión revisada de la CSE. A efectos de la protección de los DESC y específicamente del derecho a un nivel de vida adecuado, las consecuencias son innegables. Ahora bien, nuestro país está obligado al cumplimiento de la Carta original de 1961 y a sus preceptos y, entre otros, a los artículos relacionados con la protección económica y social que hemos enumerado.

\subsection{Herramientas de protección internacional del derecho a un nivel de vida adecuado. El sistema de informes y sus pronunciamientos}

Las dos principales herramientas para velar por el cumplimiento de estos tratados internacionales en materia de DESC son los informes periódicos y los mecanismos de denuncia ante los pertinentes comités. Dentro de los mecanismos de denuncia son fundamentalmente las comunicaciones individuales ante el CDESC (con relación al PIDESC) y las reclamaciones colectivas ante el CEDS (sobre la CSE). Suponen un escalón más en la exigibilidad de los DESC y son una herramienta cuasi-judicial que, aunque no es objeto del presente trabajo, es importante siquiera mencionar para contextualizar la labor de estos comités. Sus cuestiones, interpretaciones y argumentaciones se retroalimentan en ambos instrumentos (informes y denuncias) y, por ejemplo, en Europa han sido fundamentales algunas decisiones a raíz de reclamaciones colectivas del CEDS sobre las medidas anticrisis en Grecia ${ }^{1}$ o los tres dictámenes de violación del derecho a la vivienda por comunicaciones a España, en casos de desahucios, del CDESC de Naciones Unidas ${ }^{2}$.

\footnotetext{
1 Reclamaciones 76-80/2012. Se pueden consultar en Processed complaints. https:// www.coe.int/en/web/european-social-charter/processed-complaints

2 Comunicaciones 002/2014, 005/2015 y 037/2018. Se pueden consutar en Jurisprudence (OHCHR). https://juris. ohchr.org/en/search/results?Bodies=9\&sortOrder=Date
} 
El sistema de informes periódicos es un mecanismo de evaluación de los Estados, donde los comités de expertos funcionan como mecanismos de control para examinar y evaluar los progresos realizados y las dificultades encontradas con respecto a la aplicación de esos tratados (Van Boven 1997, 12). Es un mecanismo «propio del Derecho Internacional clásico, superficial y muy respetuoso de la soberanía del Estado que, en la práctica, sin embargo, ha tenido una evolución notable» (Bou y Castillo citado por Macho 2019, 7).

En el marco de Naciones Unidas, los Estados parte deben presentar un informe inicial a la entrada en vigor de los tratados $y$, posteriormente, seguir rindiendo cuentas cada cuatro o cinco años ante el órgano creado en virtud de cada tratado. En estos informes se deben exponer las medidas jurídicas, administrativas y judiciales adoptadas por el Estado, se deben mencionar también todos los factores o dificultades encontrados y rendir cuentas sobre la situación de los derechos. Cada órgano o comité establece el procedimiento y articula este sistema periódico, pero en general siguen unas pautas comunes. El Estado presenta su informe y antes del período de sesiones en el que se examinará oficialmente, el comité elabora una lista de cuestiones (omitidas en el informe anterior o consideradas necesarias para la evaluación) a responder por el Estado. Además, los comités reciben información de otras fuentes, sobre todo de otros organismos de Naciones Unidas y de organizaciones intergubernamentales, instituciones nacionales de derechos humanos, ONG internacionales y nacionales y grupos profesionales e instituciones académicas. La evaluación culmina con la aprobación de las observaciones y recomendaciones finales. Pero el proceso no termina ahí y los comités han comenzado a introducir procedimientos que facilitan el seguimiento eficaz de sus observaciones finales, a través de informes intermedios, de relatores o de visitas al país.

La Parte IV del PIDESC (ONU 1966) establece la obligación de los Estados de presentar informes sobre el respeto del Pacto ante el Comité Económico y Social; posteriormente ante su comité, el CDESC (creado en virtud de la Resolución ECOSOC 1985/17 del 28 de mayo de 1985). Así mismo, su Observación General n. ${ }^{\circ} 1$ (CDESC 1989), establece su forma y objetivos, y a partir de ambas referencias se ha venido concretando el proceso y, sobre todo, las obligaciones de información de los Estados. Después de un primer informe en los dos años siguientes a la entrada en vigor del PIDESC, los Estados parte deben seguir presentando informes periódicos cada 5 años.

Aparte de estos informes específicos del PIDESC (y de otros tratados), desde 2006 cada Estado miembro de Naciones Unidas 
se debe someter a un proceso similar para rendir cuentas sobre el cumplimiento de la DUDH, y en general, del Sistema de Derechos Humanos, denominado Examen Periódico Universal (EPU), por ciclos (cada cuatro años y medio), ante el Consejo de Derechos Humanos (establecido en 2006 por la Resolución 60/251 de la Asamblea General (A/60/L.48)). Este Consejo es un organismo intergubernamental compuesto por 47 Estados que para el EPU trabaja a través de Grupos de Trabajo. Actualmente se está desarrollando el Tercer Ciclo 20172021.

En Europa, la Parte IV de la CSE establece su sistema de informes y su marco general y específicamente la obligación de remitir al Secretario General de forma bienal un informe sobre la aplicación de las disposiciones aceptadas ${ }^{3}$ de la Parte II (art. 21) (Consejo de Europa 1961). El sistema se ha ido desarrollando a través de su Protocolo Adicional (Consejo de Europa 1991) y de la decisión de 2006 del Comité de Ministros sobre la dinámica de presentación y la decisión de 2008, sobre el contenido de los informes a la Carta original y a la Revisada. Así, en 2006 se establece que los Estados deben presentar cada año sus informes antes del 31 de octubre, sobre una de las cuatro áreas de derechos (Grupo 1: empleo, formación e igualdad de oportunidades; Grupo 2: salud, seguridad y protección social; Grupo 3: derechos laborales, y Grupo 4: infancia, familia y migración), de forma que cada grupo es evaluado cada cuatro años (Consejo de Europa 2006). En 2008 se concreta lo que los Estados parte deben aportar sobre cada artículo: marco legal, políticas, medias e información muy concreta para evaluar la eficacia de todo ello, tal que datos, estadísticas o indicadores (Consejo de Europa 2008a y 2008b).

Posteriormente, en el año 2014 se establece una nueva revisión de este sistema de informes: aquellos países que han aceptado el sistema de denuncias colectivas (en base al Protocolo Adicional a la CSE de 1995) podrán acogerse a un nuevo sistema simplificado. Este nuevo sistema parte de informes bianuales y en función del número de denuncias de cada país. Así cada año presenta informe un grupo de países $(A \circ B$ ) en función de un calendario establecido (Consejo de Europa 2014). De esta forma, se establecen dos tipos de informe en función de la adhesión del Estado al sistema de reclamaciones. En líneas generales, el objeto de estos informes es que el Estado de cuenta periódicamente de la forma en que la legislación nacional se adapta a

3 Recordemos que la CSE establece un menú de artículos a aceptar por parte de los Estados. 
la Carta, pero también sobre los esfuerzos realizados y los resultados. Los informes son analizados por el CEDS que emite su resolución de conformidad o no conformidad de cada derecho y artículo con la Carta y a partir de sus conclusiones, el Comité de Ministros elabora sus resoluciones y recomendaciones a los Estados parte.

De esta forma, los comités y órganos se pronuncian, entre otros, a través de los dictámenes (de los mecanismos de denuncia) y de las conclusiones, recomendaciones y observaciones a los informes. Los dictámenes, siguiendo la doctrina del Derecho Internacional Público y a pesar de la tradicional negativa a reconocerlos por parte de los órganos del Estado, tienen efectos jurídicos internos (Fernández de Casadevante 2020, 10). Pero hemos tenido que esperar hasta 2018 para un caso histórico de incorporación de dictámenes a la jurisprudencia interna: la Sentencia del Tribunal Supremo, STS 2747/20184, que por primera vez reconoce el carácter vinculante de un dictamen de un órgano de un tratado internacional, concretamente del Comité para la Eliminación de la Discriminación Contra la Mujer (CEDAW). En 2003, después de repetidas agresiones y denuncias por malos tratos, un hombre asesinó a su hija durante un régimen de visitas. En 2012 y tras agotar los recursos judiciales en España, el Dictamen de la CEDAW 47/2012 declara vulnerados los derechos de la autora y su hija fallecida en virtud de los art. 2. a), b), c), d), e) y f); 5 a) y 16, párrafo 1 d) de la CEDAW y recomienda al Estado otorgar una indemnización integral y proporcional al daño e investigar de forma exhaustiva e imparcial los actos negligentes del Estado. La STS de 2018, obliga al cumplimiento de este Dictamen y la consiguiente responsabilidad patrimonial del Estado. De esta forma, esperemos que esta sentencia sea un punto de inflexión para el reconocimiento del carácter vinculante de los dictámenes de los comités y órganos internacionales y su efectivo complimiento.

Como señala Fernández de Casadevante (2020), los dictámenes de los comités son derecho español y su incumplimiento supone también el incumplimiento de la Constitución; de la aplicación de los art. 1.1, 9.2, 9.3, 10.2, 96 y 95 y de las obligaciones internacionales inherentes tanto a la ratificación de España de los tratados internacionales que establecen los comités como a las declaraciones de aceptación de su competencia realizada por nuestro país.

\footnotetext{
${ }^{4}$ De 17 de julio de 2018. Roj: STS 2747/2018 - ECLI: ES:TS:2018:2747. Consultado en: http://www.poderjudicial.es/search/documento/TS/8457097/Responsabilidad\%20 patrimonial/20180723
} 
Pero España también debe cumplir los pronunciamientos de los informes periódicos. Es inadmisible que los dictámenes de los comités y todas sus actividades de control sean repetidamente minusvaloradas, calificándolas como meras decisiones políticas, cuando estamos ante decisiones que tienen como referencia las disposiciones jurídicas contenidas en los tratados internacionales y han sido tomadas por órganos autorizados para ello (Faleh 2020, 93). O de otra forma, tienen el mismo valor jurídico que los tratados y deben ser aplicadas de buena fe por los Estados (Villán 2020, 121). Y, aunque en la práctica las políticas públicas y las mejoras legislativas en la mayoría de los casos no los han venido incorporando, esto no quiere decir que debamos renunciar a considerarlos, entenderlos y, de nuevo, reclamarlos como obligatorios.

\subsection{Los informes periódicos de España}

España se somete al Tercer EPU el miércoles 22 de enero de $2020^{5}$. Anteriormente el 21 de enero de 2015 nuestro país se sometió al Segundo Ciclo, para el cual el Consejo de Derechos Humanos contaba con el informe de España (CDH 2015a), con una recopilación del ACNUDH sobre los informes de los órganos de los tratados y los procedimientos especiales, y un resumen de las comunicaciones presentadas por ONG y partes interesadas. Analizado todo ello, el Grupo de Trabajo aprueba el informe final (CDH 2015b) donde se plasman, entre otras cuestiones, las 189 recomendaciones realizadas a España. De todas ellas, nuestro país aceptó 169 (cinco de ellas parcialmente) y «tomó nota» de veinte (CDH 2015c). Casi todas las recomendaciones rechazadas se relacionan con la ratificación de instrumentos internacionales y especialmente la Convención Internacional sobre la Protección de los Derechos de todos los trabajadores migratorios y de sus familiares de 1990 y que, efectivamente, España no ha ratificado; o con medidas legislativas para proteger a los menores no acompañados, entre otras. Por otro lado, acepta todas las recomendaciones que afectan directamente a DESC. Además, en 2018, presentó un informe intermedio sobre la implementación de estas recomendaciones (Oficina de Derechos Humanos 2018).

5 Recién concluido el 35 periodo de sesiones (el 31 de enero de 2020), se ha publicado una primera versión no editada del resultado del EPU o Draft report of the Working Group (A/HRCMG.6/35/L.4). Pero el informe del Grupo de Trabajo aún no se ha incorporado a la Base de Datos del EPU (CDH 2020). Ha sido publicado un avance y alerta de Naciones Unidas que informa sobre los principales resultados (ONU 2020b). 
Tabla 1

\section{Esquema de las obligaciones de España y los informes periódicos presentados relacionados con los DESC}

\begin{tabular}{|c|c|c|c|c|c|c|}
\hline & Tratado & $\begin{array}{l}\text { Comité / } \\
\text { Organismo }\end{array}$ & $\begin{array}{l}\text { Sistema de } \\
\text { Informes }\end{array}$ & \multicolumn{3}{|c|}{ Presentados } \\
\hline \multirow[t]{2}{*}{ ONU } & $\begin{array}{l}\text { Declaración } \\
\text { Universal y } \\
\text { Derechos } \\
\text { Humanos en } \\
\text { general }\end{array}$ & $\begin{array}{l}\text { Consejo de } \\
\text { Derechos } \\
\text { Humanos }\end{array}$ & $\begin{array}{l}\text { Examen } \\
\text { Periódico } \\
\text { Universal } \\
\text { (EPU). } \\
\text { Cada 4-5 } \\
\text { años. }\end{array}$ & \multicolumn{3}{|c|}{$\begin{array}{l}3 \text { Ciclos. Informes } \\
\text { de: } \\
2010 \\
2015 \\
2020^{*}\end{array}$} \\
\hline & $\begin{array}{l}\text { Pacto } \\
\text { Internacional } \\
\text { de DESC } \\
\text { (PIDESC) }\end{array}$ & $\begin{array}{l}\text { Comité de } \\
\text { Derechos } \\
\text { Económicos, } \\
\text { Sociales y } \\
\text { Culturales } \\
\text { (CDESC) }\end{array}$ & $\begin{array}{l}\text { Informes } \\
\text { periódicos } \\
\text { al PIDESC. } \\
\text { Cada } 5 \\
\text { años }\end{array}$ & \multicolumn{3}{|c|}{$\begin{array}{l}6 \text { informes: el } \\
\text { último en } 2017\end{array}$} \\
\hline \multirow{7}{*}{$\begin{array}{l}\text { Consejo de } \\
\text { Europa } \\
\text { (CE) }\end{array}$} & \multirow{7}{*}{$\begin{array}{l}\text { Carta Social } \\
\text { Europea } \\
\text { (CSE) }\end{array}$} & \multirow{7}{*}{$\begin{array}{l}\text { Comité } \\
\text { Europeo de } \\
\text { Derechos } \\
\text { Sociales } \\
\text { (CEDS) }\end{array}$} & \multirow{7}{*}{$\begin{array}{l}\text { Informes a } \\
\text { la CSE. } \\
\text { Sobre un } \\
\text { Grupo } \\
\text { cada año } \\
\text { (4 Grupos) }\end{array}$} & \multicolumn{3}{|c|}{$\begin{array}{l}32 \text { informes: los } \\
\text { últimos }\end{array}$} \\
\hline & & & & N. ${ }^{\circ}$ & Año & Grupo \\
\hline & & & & $32 *$ & 2019 & 1 \\
\hline & & & & 31 & 2018 & $4^{* *}$ \\
\hline & & & & 30 & 2017 & 3 \\
\hline & & & & 29 & 2016 & $2 * *$ \\
\hline & & & & 28 & 2015 & 1 \\
\hline
\end{tabular}

Fuente: Elaboración propia.

* Las conclusiones / observaciones finales del Comité / Consejo aún no son accesibles.

** Incluyen artículos directamente relacionados con un nivel de vida adecuado y a la protección económica y social.

Respecto a los informes periódicos al PIDESC, para el último del año 2017 se presentaron previamente, en 2016, 7 informes a las consultas previas, casi todos de ONG (más un informe del Defensor del Pueblo), sobre cuestiones concretas que debería aportar España en su informe. Con esta información y las recomendaciones 
del anterior informe de 2009, el Comité elabora una lista de 26 cuestiones previas (CDESC 2016). En septiembre de 2017, España presenta su documento (CDESC 2017). Éste se divide en dos apartados: cuestiones de particular importancia y aplicación en curso del PIDESC. El esquema se compone de veinticinco puntos que responden a las cuestiones previas planteadas; pero debemos pensar que el Estado también debe rendir cuentas sobre las recomendaciones realizadas por el CDESC al informe anterior. El Comité examinó todo ello y en marzo de 2018 aprobó las observaciones finales (CDESC 2018) y, posteriormente, España presenta un informe sobre el seguimiento de estas observaciones finales (CDESC 2019).

En el marco del Consejo de Europa y la CSE, hay que recordar que España no ha ratificado la CSE revisada, ni el Protocolo Facultativo de 1995 sobre reclamaciones colectivas, por lo que no se puede acoger al sistema simplificado de informes. Debe seguir el procedimiento originario y presentar informes anuales por el sistema de Grupos. Como se observa en la tabla 1, entre 2015 y 2018 se cubrieron los cuatro Grupos, comenzando en 2019 de nuevo por el Grupo 1. El último informe que incluye los art. 12 a 14 (derecho a la seguridad y asistencia social) es el $n .{ }^{\circ} 29$ de 2016; y los art. 16 y 17 (sobre derecho de la familia, las madres y los niños a la protección social, jurídica y económica) es el n. ${ }^{\circ} 31$ de 2018. Ambos informes (n. ${ }^{\circ} 29$ y 31), fueron acompañados de información paralela de organizaciones sindicales $y$, un año después, sometidos a examen, emitiendo el Comité sus conclusiones XXI-2 (2017) (Consejo de Europa 2020a) y conclusiones XX-4 (2019) (Consejo de Europa 2020b), respectivamente.

\section{Recomendaciones y conclusiones de los órganos internacionales a los últimos informes de España}

La tabla 2 plasma las recomendaciones y conclusiones a los informes de España analizadas en este trabajo. Proceden de los tres órganos fundamentales de derechos humanos y de DESC internacionales, cubren el periodo desde 2014 y se centran específicamente en los artículos directamente relacionados con el derecho a un nivel adecuado y a la protección económica y social: el artículo 25.1 de la DUDH, artículos 11 y 9 del PIDESC y artículos 12, $13,14,16$ y 17 de la CSE. 
Tabla 2

\section{Conclusiones y recomendaciones analizadas}

\begin{tabular}{|c|c|c|c|c|}
\hline Informe & Tratado & Artículos & $\begin{array}{l}\text { Comité/ } \\
\text { Organismo }\end{array}$ & $\begin{array}{l}\text { Recomendaciones/ } \\
\text { Conclusiones }\end{array}$ \\
\hline $\begin{array}{l}2015 \\
\text { EPU }\end{array}$ & $\begin{array}{l}\text { Declaración } \\
\text { Universal } \\
\text { DDHH } \\
\text { (DDHH en } \\
\text { general) } \\
\end{array}$ & $\begin{array}{l}25.1 \\
\text { (y áreas } \\
\text { relacionadas) }\end{array}$ & $\begin{array}{l}\text { Consejo de } \\
\text { Derechos } \\
\text { Humanos }\end{array}$ & $\begin{array}{l}\text { A/HRC/29/8 } \\
(2015)^{\star *} \\
11 \text { relacionadas } \\
\text { con áreas nivel } \\
\text { vida adecuado }\end{array}$ \\
\hline $\begin{array}{l}2016 \\
\text { N. }{ }^{\circ} 29 \\
\text { Grupo } 2\end{array}$ & $\begin{array}{l}\text { Carta Social } \\
\text { Europea } \\
\text { (CSE) }\end{array}$ & $\begin{array}{l}12,13 \text { y } 14 \\
\text { (Grupo 2) }\end{array}$ & $\begin{array}{l}\text { Comité } \\
\text { Europeo de } \\
\text { Derechos } \\
\text { Sociales } \\
\text { (CEDS) }\end{array}$ & $\begin{array}{l}\text { Conclusiones } \\
\text { XXI-2 (2017)* } \\
12 \text { referidas a } \\
\text { Art. } 12,13 \text { y } 14\end{array}$ \\
\hline $\begin{array}{l}2017 \\
\text { Al PIDESC }\end{array}$ & $\begin{array}{l}\text { Pacto } \\
\text { Internacional } \\
\text { de DESC } \\
\text { (PIDESC) }\end{array}$ & $\begin{array}{l}11 \\
9\end{array}$ & $\begin{array}{l}\text { Comité de } \\
\text { Derechos } \\
\text { Económicos, } \\
\text { Sociales y } \\
\text { Culturales } \\
\text { (CDESC) }\end{array}$ & $\begin{array}{l}\text { E/C. 12/ESP/6 } \\
(2018) * * * \\
4 \text { relacionas con } \\
\text { Art. } 11 \text { y } 9\end{array}$ \\
\hline $\begin{array}{l}2018 \\
\text { N. }{ }^{\circ} 31 \\
\text { Grupo } 4\end{array}$ & $\begin{array}{l}\text { Carta Social } \\
\text { Europea } \\
\text { (CSE) }\end{array}$ & 16 y 17 & $\begin{array}{l}\text { Carta Social } \\
\text { Europea } \\
\text { (CSE) }\end{array}$ & $\begin{array}{l}\text { Conclusiones } \\
\text { XXI-4 } \\
(2019) \star \star \star \star \\
2 \text { referidas a } \\
\text { Art. } 16 \text { y } 17\end{array}$ \\
\hline
\end{tabular}

Fuente: Elaboración propia.

* Fuente conclusiones: CE 2020b.

** Fuente observaciones y recomendaciones: CDH 2020.

*** Fuente observaciones y recomendaciones: CDESC 2020b.

$\star \star \star \star$ Fuente conclusiones: CE 2020c.

2.1. Recomendaciones del Consejo de Derechos Humanos de Naciones Unidas. EPU de 2015

Para el último EPU de 2020 podemos contar con un resultado preliminar del Grupo de Trabajo, según el cual se han realizado a España 275 recomendaciones para mejorar los derechos humanos. Entre otras cuestiones, distintos Estados proponen fortalecer las políticas de lucha contra el desempleo y promover el empleo decente, especialmente entre los jóvenes y la población romaní, y adoptar 
las medidas necesarias para ampliar la cobertura de las pensiones. Con respecto al problema del acceso a la vivienda y los desalojos, recomiendan a España promover la construcción generalizada de viviendas sociales para las familias vulnerables y adoptar un marco legislativo relativo al derecho a la vivienda para garantizar que las personas desalojadas tengan acceso a una indemnización u otra vivienda decente, que se les informe de la decisión de desalojo y que tengan acceso a un recurso judicial efectivo (ONU 2020b).

Centrándonos en el EPU anterior de 2015, vemos que, de las 189 recomendaciones a España, once estaban relacionadas directamente con el derecho a un nivel de vida adecuado y fueron aceptadas por España: siete (125 a 130 y 132) pertenecen al área sobre medidas para implementar los DESC, dos (124 y 123) sobre el propio art. 25.1 y otras dos (133 y 134) sobre el derecho a la vivienda. Sobre la primera área, preocupa al Consejo las medidas tomadas para mitigar los efectos de la crisis económica entre los sectores más vulnerables en general y, sobre todo, el impacto de las medidas de austeridad. En una línea similar, se enuncian las dos recomendaciones directamente relacionadas con el derecho a un nivel de vida adecuado, encaminadas a mitigar los efectos de la crisis sobre las personas en riesgo de exclusión, sobre todo los niños y las niñas. Respecto al derecho a la vivienda, se centran en la necesidad de implementar medidas que regulen y mitiguen los desalojos (12 y 13) (CDH 2015d).

\subsection{Recomendaciones del CDESC de Naciones Unidas. Informe al PIDESC de 2017}

Un año antes del informe de 2017, el Comité en la lista de cuestiones previas pedía a España que aportase, entre otras, información sobre: la garantía de que los niveles de protección fueran reforzados con la recuperación económica (n. $\left.{ }^{\circ} 4\right)$, sobre los niveles de pobreza y las medidas tomadas $\left(n .^{\circ} 5\right)$, sobre medidas para evitar desahucios y garantizar la seguridad jurídica ( $\left.n .^{\circ} 6\right)$, medidas para ajustar el salario mínimo a un nivel de vida adecuado (.$^{\circ}{ }^{16}$ ), medias para garantizar la alimentación adecuada y asequible (n. $\left.{ }^{\circ} 18\right)$, sobre vivienda social y cambios en el modelo de vivienda (n. $\left.{ }^{\circ} 19\right)$ y medidas para paliar el sinhogarismo ( . $\left.^{\circ} 20\right)$ (CDESC 2016). Tras el informe de España y la información paralela de la sociedad civil, el Comité elabora sus observaciones y 20 recomendaciones específicas (y cinco genéricas) (CDESC 2018). De todas ellas, cuatro hacen referencia directa a los art. 9 y 11 del PIDESC; y de estas cuatro casi todas (sobre 
todo relacionadas con la seguridad social, el derecho a una vivienda adecuada y los desahucios) ya habían sido motivo de preocupación en el ciclo anterior (CDESC 2012).

Respecto al art. 9 sobre el derecho a la seguridad social, el Comité llama la atención sobre el déficit del sistema de pensiones, la baja tasa de cobertura de las prestaciones no contributivas y, en general, sobre la insuficiencia de las cuantías de las prestaciones para garantizar un nivel de vida adecuado. Y para ello, recomienda a España: adoptar medidas, contando con los actores sociales (y en especial los sindicatos), para asegurar la sostenibilidad del sistema de pensiones, «intensificar los esfuerzos» para ampliar la cobertura del sistema de seguridad social a los sectores más desfavorecidos, así como aumentar las prestaciones para garantizar un nivel de vida adecuado a los beneficiarios. En este sentido alienta a establecer un sistema eficaz y transparente (CDESC 2018, párrafos 30 y 31).

Con relación al art. 11, sobre el derecho a un nivel de vida adecuado, el Comité se muestra preocupado sobre la alta tasa de pobreza y exclusión social («para un país con el nivel de desarrollo del Estado parte»), sobre todo entre los jóvenes, mujeres, migrantes y población con bajo nivel educativo; así como las importantes diferencias por Comunidades Autónomas y el alto riesgo de pobreza infantil. Por ello, recomienda a España que agilice la elaboración y adopción de la estrategia nacional de prevención y lucha contra la pobreza y exclusión 2018-20206. Una estrategia que debería tener en cuenta especialmente a la infancia, tener un enfoque de derechos humanos y suficientes recursos, en función de las disparidades territoriales (CDESC 2018, párrafos 33 y 34).

El Comité realiza así mismo dos recomendaciones sobre el derecho a la vivienda (art. 11). En primer lugar, llama la atención sobre el impacto de las medidas de austeridad en el deterioro del derecho, sobre todo de los sectores más vulnerables: el número insuficiente de vivienda social, el alto precio de la vivienda, falta de seguridad de la tenencia y el alto número de hogares con vivienda en condiciones no adecuadas o sin hogar. Para ello, el Comité recomienda: aumentar la vivienda social, sobre todo para las personas sin hogar o de bajos ingresos, jóvenes, mujeres o personas son discapacidad, tomar medidas en el modelo de mercado para facilitar el acceso a la vivienda, revisar la legislación sobre arrendamientos para garantizar la tenencia y los

6 La Estrategia Nacional de Prevención y Lucha contra la Pobreza y la Exclusión Social fue aprobada en marzo de 2019 para el período 2019-2023. Véase: Ministerio de Sanidad, Consumo y Bienestar Social. https://www.mscbs.gob.es/ 
mecanismos judiciales para la protección de derecho a la vivienda adecuada, tomar medidas para mejorar la calidad de la vivienda y asegurar la provisión adecuada de centros de acogida en las Comunidades Autónomas (CDESC 2018, párrafos 35 y 36).

En segundo lugar, de especial preocupación para el CDESC es la cuestión de los desahucios. Reconociendo los avances, pone el foco sobre la falta de un marco legislativo adecuado que determine las debidas garantías legales y procesales de las personas afectadas. Para ello, recomienda adoptar un marco legislativo que regule la cuestión, asegurando la compensación o vivienda alternativa, plazos suficientes y recursos judiciales efectivos; así como un protocolo de coordinación entre los tribunales y los servicios sociales (CDESC 2018, párrafos 37 y 38).

Sobre desahucios, es indicador de la alarma que trasmite el Comité el hecho de que, en estas conclusiones finales, inste a España, entre otros, a presentar informe de seguimiento (en los 18 meses siguientes a la adopción de las observaciones) sobre ello y sobre el impacto de las medidas de austeridad (CDESC 2018, párrafo 55). Efectivamente, en 2019 España presenta dicho informe donde da cuenta sobre las reformas legales de 2018 y 2019: la Ley 5/2018, de 11 de junio, de modificación de la Ley 1/2000, de 7 de enero, de Enjuiciamiento Civil, con relación a la ocupación ilegal de viviendas y el Real Decreto-ley 7/2019, de 1 de marzo, de medidas urgentes en materia de vivienda y alquiler (CDESC 2019, párrafos 7-15).

Además de estos cuatro puntos concretos, el Comité también llama la atención, al igual que en la evaluación anterior de 2012, sobre el impacto de las medidas de austeridad, su temporalidad y proporcionalidad y afectación a los sectores más vulnerables (CDESC 2018, párrafos 13 y 14), las disparidades regionales en el disfrute de los DESC (párrafos 11 y 12) y la garantía del derecho a un nivel de vida adecuado de los migrantes, solicitantes de asilo y refugiados (párrafos 39 y 40).

\subsection{Conclusiones del CEDS del Consejo de Europa. Informes $n .^{\circ} 29$ de 2016 y n. ${ }^{\circ} 31$ de 2018}

Como se observa en la tabla 3, la situación de España se ajusta a lo dispuesto en la Carta en siete artículos; en otros cuatro, el Comité queda a la espera de recibir información, en un caso el Comité aplaza su conclusión a la espera de recibir información y en otros cuatro, el dictamen es de no conformidad. 
Tabla 3

\section{Conclusiones del CEDS a los informes de España de 2016 y 2018, relacionadas con los art. 16, 17, 12, 13 y 14 de la CSE}

\begin{tabular}{|c|c|c|c|}
\hline Informe & Artículo & Conclusión & $\begin{array}{c}\text { Dictamen } \\
\text { Conformidad con la Carta }\end{array}$ \\
\hline \multirow{10}{*}{$\begin{array}{l}2016 n .^{\circ} 29 \\
\text { Grupo } 2\end{array}$} & 12.1 & XXI-2/def/ESP/12/1/EN & $\mathrm{NO}$ \\
\hline & 12.2 & XXI-2/def/ESP/12/2/EN & $\mathrm{SI}$ \\
\hline & 12.3 & XXI-2/def/ESP/12/3/EN & SI (pendiente de información) \\
\hline & 12.4 & XXI-2/def/ESP/12/4/EN & $\mathrm{NO}$ \\
\hline & 13.1 & XXI-2/def/ESP/13/1/EN & $\mathrm{NO}$ \\
\hline & 13.2 & XXI-2/def/ESP/13/2/EN & SI (pendiente de información) \\
\hline & 13.3 & XXI-2/def/ESP/13/3/EN & SI (pendiente de información) \\
\hline & 13.4 & XXI-2/def/ESP/13/4/EN & $\mathrm{SI}$ \\
\hline & 14.1 & XXI-2/def/ESP/14/1/EN & SI (pendiente de información) \\
\hline & 14.2 & XXI-2/def/ESP/14/2/EN & $\mathrm{SI}$ \\
\hline \multirow{2}{*}{$\begin{array}{l}2018 \text { n. } .^{\circ} 31 \\
\text { Grupo } 4\end{array}$} & 16 & $X X-4(2019)$ & $\mathrm{NO}$ \\
\hline & 17 & $X X-4(2019)$ & Aplazada \\
\hline
\end{tabular}

Fuente: Elaboración propia a partir de CE 2020b y CE 2020c.

En el caso de las conclusiones de conformidad: el Comité entiende que España cumple la CSE con relación al mantenimiento de un sistema de seguridad social según el estándar del Convenio n. ${ }^{\circ} 102$ de la OIT (art. 12.2), el derecho a la asistencia social y médica de emergencia para no residentes (art. 13.4) y la participación ciudadana y de la sociedad civil en el establecimiento y mantenimiento de los servicios sociales (art. 14.2).

Sobre las conclusiones de conformidad, pero con carencias de información, el análisis es el siguiente. En relación con el desarrollo del sistema de seguridad social y el esfuerzo por elevar progresivamente el nivel del régimen de seguridad social (art. 12.3), el Comité toma nota de medidas restrictivas en las pensiones (cálculo de revalorización o criterios de cotización), y en las prestaciones de desempleo asistenciales. Queda a la espera de recibir datos sobre otras medidas 
con impacto en la cobertura personal y niveles de prestaciones, tales como las revisiones para 2016 de la legislación general de seguridad social (RDL 8/2015).

El Comité también queda pendiente de que España aporte información sobre la prohibición de la discriminación contra las personas que reciben asistencia social o médica en el ejercicio de sus derechos políticos o sociales (art. 13.2), sobre los mecanismos para garantizar que las personas necesitadas puedan recibir ayuda y servicios de asesoramiento personal gratuitos (para el pleno acceso a la asistencia social y médica) y, así mismo, si están geográficamente bien distribuidos (art. 13.3) y sobre el desarrollo de los instrumentos específicos establecidos para promover la calidad de los servicios sociales en todas las Comunidades Autónomas (art. 14.1).

Sobre el art. 17, el Comité aplaza su decisión a falta de recibir información, especialmente sobre niños y niñas en situación vulnerable: sobre medidas para garantizar el registro e inscripción de niños y niñas migrantes, número de niños y niñas en instituciones (en línea con la preocupación y las observaciones del Comité de los Derechos del Niño de las Naciones Unidas, sobre el quinto y sexto informe periódico de España), sobre menores en conflicto con la ley y sobre la asistencia y protección de los migrantes menores no acompañados, especialmente en casos de abusos y explotación. Mención aparte merece la argumentación del Comité sobre la pobreza infantil en España. A diferencia de las conclusiones anteriores al informe n. 27 de 2014, en esta ocasión, el Comité habla explícitamente de ello y relaciona directamente este art. 17 con la pobreza infantil. Llama la atención sobre los altos niveles en España (por encima de la media de la UE) y pide datos sobre ello e información sobre las medidas tomadas para paliarlo, y contra la discriminación de grupos vulnerables (niños y niñas romanís o con discapacidad).

Y finalmente, el Comité considera que la situación en España no es conforme a cuatro artículos de la CSE: Sobre el art. 16, entiende que los subsidios familiares son insuficientes. Al igual que en las conclusiones anteriores (Conclusiones XX-4 (2015)), el nivel de los subsidios con hijos a cargo sigue estando muy por debajo del nivel de referencia (40\%-50\% de la mediana del ingreso equivalente respecto al umbral de pobreza) y a pesar de medidas como el Real Decreto Legislativo 8/2019, los niveles de protección familiares no son adecuados. De hecho, el Comité entiende que la prestación del sistema de protección social español por cada hijo a cargo, que es recibido por casi 800.000 familias y congelado a 291 euros por 
año, es claramente inadecuado y no hace nada para resolver el grave problema de la pobreza infantil en España, que se ve agravada por la crisis económica. Además, pide información a España, entre otros, sobre la evaluación del Plan Integral de Apoyo a la Familia 2015-2017 y sobre el número y cobertura de plazas públicas de guardería. Y de nuevo, en esta Conclusión se solicita a España información sobre el derecho a la vivienda y sus salvaguardas y protección judicial; concretamente sobre el impacto del Real Decreto 106/2018 de 9 de marzo 2018 que establece el reglamento del Plan Nacional de Vivienda 2018-2021 en las familias más vulnerables (romanís y monoparentales), sobre el marco legislativo y protección jurídica en casos de desalojos, cifras actualizadas y jurisprudencia sobre proporcionalidad del desalojo; así como número y cobertura de vivienda social y sobre prevalencia de infravivienda. El Comité menciona y toma como referencia las conclusiones del CDESC de Naciones Unidas sobre el Sexto informe de España al PIDESC.

Con relación al art. 12.1 (existencia y mantenimiento de un sistema de seguridad social), el principal motivo de no conformidad es la insuficiencia de la prestación por desempleo en caso de no tener familia a cargo, que en 2015 era menor del 40\% de la renta mediana. $Y$, de nuevo, el Comité pide información a España sobre la cobertura de las prestaciones de sustitución de ingresos, sobre complementos a las prestaciones mínimas por enfermedad durante los primeros veinte días de incapacidad y sobre la duración de las prestaciones por desempleo.

El art. 12.4 se refiere a la seguridad social de las personas desplazadas de otros Estados parte y la situación de España es de no conformidad, dado que no se garantiza la igualdad de trato en el acceso a subsidios familiares a las familias de otros Estados parte (especialmente con los que España no ha firmado acuerdos bilaterales que garanticen la igualdad de trato en materia de seguridad social) y que el requisito de residencia de diez años para el acceso a una pensión de jubilación no contributiva es excesivo.

Respecto al art. 13.1 (asistencia social y médica adecuada a cada persona en situación de necesidad), el Comité considera que el acceso y la cobertura de los ingresos mínimos garantizados no se ajustan a la Carta dado que en la mayoría de las Comunidades Autónomas la renta mínima garantizada es inferior al $50 \%$ de la renta mediana y se necesita un tiempo de residencia mínimo. Además, el Comité se apoya en otros dos motivos: el requisito de edad mínima de veinticinco años y el tiempo limitado de la renta mínima (y el Comité considera que se debe mantener mientras persista la situación de necesidad). 
Pero muchas de estas cuestiones y pronunciamientos del CEDS venían ya de años atrás. En el análisis que realiza Jimena Quesada (2014, 180-182) del anterior examen del Grupo 2 (art. 12 a 14) en 2013 (informe n. 25 de 2012), vemos que el Comité se pronunció en términos similares y determinó que España no actuaba conforme a la Carta en los mismos art. 12.1, 12.4, y 13.1. Y con argumentos casi idénticos: la insuficiencia de las prestaciones por enfermedad (art. 12.1), la falta de garantía de la igualdad de trato de los nacionales de todos los demás Estados partes en materia de derechos a la seguridad social y de acceso a las prestaciones familiares (art. 12.4) y la subordinación de la renta mínima a la condición de residencia (que oscila entre seis meses y tres años según las Comunidades Autónomas), a la edad (25 años), por tiempo limitado (no durante toda la situación de necesidad) y por debajo del umbral de pobreza (fijado en el $50 \%$ de la renta mediana ajustada calculada por Eurostat sobre la base del umbral del riesgo de pobreza) (art. 13.1).

Sobre este punto es importante mencionar la delimitación que hace el CEDS de la adecuación de las cuantías mínimas de prestación por cualquier motivo como un ingreso que no debe ser inferior al valor umbral de Eurostat de riesgo de pobreza. Si las tasas mínimas se reducen entre el $40 \%$ y el $50 \%$ de la mediana del ingreso equivalente, se pueden tener en cuenta los beneficios suplementarios. Pero para los beneficios que caen por debajo del umbral del $40 \%$, el Comité sostiene que son «manifiestamente inadecuados» (Leppik 2016). Incluso, como de nuevo señala Jimena Quesada $(2014,181)$, el baremo usado por el CEDS, del $60 \%$ de la renta mediana ajustada convencionalmente admitido por Eurostat, es la línea identificadora de las situaciones de «baja renta». Efectivamente, este punto de referencia del umbral de pobreza para las prestaciones viene siendo clave en las conclusiones del Comité; en este ciclo de informes de 2017 pone el énfasis, además de en los altos niveles de pobreza en Europa, en el incumplimiento de la Carta en base a los niveles de protección por debajo de este indicador (Consejo de Europa 2018).

\section{Conclusiones}

España tiene obligaciones internacionales en materia de derechos humanos y de DESC. Partiendo de la DUDH, nuestro país ratificó en 1977 el PIDESC y en 1980 la CSE y en base a estos tres tratados nuestro país debe promocionar, respetar y cumplir el derecho a un nivel de vida adecuado y a la protección económica y social; de forma 
que la pobreza es más que la falta de ingresos, supone la violación de derechos humanos. Pero España no ha ratificado la CSE Revisada de 1996 que incorpora explícitamente el derecho a la protección contra la pobreza y la exclusión social (art. 30) y a la vivienda (art. 31), tampoco el Protocolo Facultativo a la CSE de 1995 que supondría que los ciudadanos españoles, a través de entidades, pudiéramos reclamar la violación de la CSE. Es inexplicable que nuestro país no forme parte de estas dos referencias ineludibles en Europa para la garantía, defensa y exigibilidad de los DESC. Pero en espera de estas adhesiones, afortunadamente, el CEDS, como hemos visto, ha hecho una interpretación extensiva de la CSE original para la garantía del derecho a un nivel de vida adecuado y a la protección económica y social.

Así mismo, debe rendir cuentas ante los órganos y comités de estos tratados a través de los informes periódicos. Desde 2015 España se ha sometido a cuatro evaluaciones sobre el cumplimiento del derecho a un nivel de vida adecuado y a la protección económica y social, con los consiguientes pronunciamientos, de cuyo análisis podemos extraer tres conclusiones principales.

Primera. Es motivo de preocupación los altos índices de pobreza y de pobreza infantil en España; así como la desigual recuperación económica y el impacto de las medidas de austeridad: por su temporalidad, proporcionalidad y afectación a los sectores más vulnerables. El CDESC insta a España a poner en marcha una clara estrategia contra la pobreza y el CEDS reclama información sobre las medidas concretas tomadas para paliar la pobreza infantil y sobre la evaluación del Plan Integral de Apoyo a la Familia 2015-2017. Y la conclusión es clara: la discrepancia entre el nivel económico de nuestro país y los altos índices de pobreza es incompatible con el efectivo cumplimiento del derecho a un nivel de vida adecuado.

Segundo. Son fundamentales las políticas sociales y las iniciativas legislativas encaminadas a paliar dos cuestiones esenciales sobre el derecho a la vivienda. El aumento de la vivienda social y, sobre todo, la regulación de los desalojos y desahucios para el acceso a vivienda alternativa y garantías y recursos judiciales. A pesar de los esfuerzos realizados, el CEDS pide información al respecto a España y se hace eco de las observaciones del CDESC de Naciones Unidas. Efectivamente este Comité ha sido muy riguroso respecto al respeto del derecho a la vivienda en España, en el contexto de su importante labor en la defensa de este derecho a través de las comunicaciones individuales, donde ha determinado en tres ocasiones que España ha violado el art. 11 del PIDESC. 
Tercero. Otra causa recurrente de incumplimiento del derecho a un nivel de vida adecuado es el acceso, la cobertura y, sobre todo, la cuantía de las prestaciones en España. Es motivo de preocupación para el CDESC (especialmente las no contributivas) y argumento de no conformidad con la CSE porque no alcanzan el mínimo requerido entre el $40-50 \%$ de la mediana de ingreso equivalente respecto al umbral de pobreza. Concretamente los subsidios familiares con hijos a cargo (incumpliendo el art. 16); prestaciones por desempleo sin familia a cargo (art. 12.1) y la renta mínima garantizada en la mayoría de las Comunidades Autónomas (art. 13.1).

En definitiva, los órganos y comités internacionales, a través de sus pronunciamientos en las evaluaciones periódicas, han delimitado medidas y objetivos muy concretos que nuestro país debe alcanzar para cumplir plenamente sus obligaciones internacionales y que efectivamente, son vinculantes para los poderes públicos, todos, independientemente de los gobiernos y las orientaciones políticas. Deben ser referencia obligada de la sociedad civil, de las políticas públicas, que no olvidemos desarrollan los principios rectores de la política social y económica (Capítulo III de la Constitución) de los que se derivan la mayoría de los DESC, y de las reformas y mejoras legislativas que garanticen estos derechos.

La lucha contra la pobreza no es una cuestión sólo de voluntad política, de decencia moral o incluso ideologías o creencias; es cuestión de derechos humanos y de obligaciones contraídas por el Estado. Y ahora más que nunca necesitamos esos referentes normativos y sus herramientas de garantía y exigibilidad para afrontar, sin terminar de salir aún de la Gran Recesión de 2014, el impacto del COVID-19. Como bien ha señalado Giuseppe Palmisano, presidente del CEDS, la crisis de COVID-19 es un recordatorio brutal de la importancia de garantizar un progreso duradero en el disfrute de los derechos sociales. Es crucial que la CSE se utilice para dar forma y analizar las decisiones durante la crisis de COVID-19. La Carta sirve como una herramienta clave para que los Estados se aseguren de que sus respuestas a la pandemia cumplen con los derechos humanos?.

7 Véase: Social rights of children, families and migrants in danger across Europe: latest annual conclusions from the European Committee of Social Rights. https://www. coe.int/en/web/european-social-charter/-/social-rights-of-children-families-and-migrantsin-danger-across-europe-latest-annual-conclusions-from-the-european-committee-ofsocial-rights 


\section{Bibliografía}

ACNUDH. 2019. Alto Comisionado de Naciones Unidas para los Derechos Humanos. Human rights dimension of poverty. Acceso 9 noviembre de 2019. https://www.ohchr.org/EN/Issues/Poverty/DimensionOfPoverty/ Pages/Index.aspx

Añón, M. ${ }^{a}$ José. 2014. "Derecho a un nivel de vida adecuado y medios de subsistencia». En El sistema universal de los derechos humanos, editado por Cristina Monereo y J. Luis Monereo, 625-637. Granada: Biblioteca Comares de Ciencia Jurídica.

Comité de Derechos Económicos, Sociales y Culturales de Naciones Unidas (Comité DESC). 1989. Observación general No 1. Presentación de informes por los Estados Partes. E/1989/22. Acceso 20 noviembre de 2019. https://tbinternet.ohchr.org/_layouts/15/TreatyBodyExternal/Countries. aspx? CountryCode=ESP\&Lang=EN

Comité DESC. 2012. Examen de los informes presentados por los Estados partes en virtud de los artículos 16 y 17 del Pacto. España. E/C.12/ESP/ CO/5. Acceso 15 septiembre de 2019. https://undocs.org/es/E/C.12/ESP/ $\mathrm{CO} / 5$

Comité DESC. 2016. Lista de cuestiones previas a la presentación del sexto informe periódico de España. E/C.12/ESP/QPR/6. Acceso 15 septiembre de 2019. https://undocs.org/es/E/C.12/ESP/QPR/6

Comité DESC. 2017. Sexto informe periódico que España debía presentar en 2017 en virtud de los artículos 16 y 17 del Pacto. E/C.12/ESP/6. Acceso 15 septiembre de 2019. https://undocs.org/es/E/C.12/ESP/6

Comité DESC. 2018. Observaciones finales sobre el sexto informe periódico de España. E/C.12/ESP/CO/6. Acceso 15 septiembre de 2019. https://undocs. org/es/E/C.12/ESP/CO/6

Comité DESC. 2019. Observaciones finales sobre el sexto informe periódico de España Adición Información recibida de España sobre el seguimiento de las observaciones finales. E/C.12/ESP/CO/6/Add.1. Acceso 30 septiembre de 2019. https://tbinternet.ohchr.org/_layouts/15/TreatyBodyExternal/ Countries.aspx?CountryCode=ESP\&Lang=EN

Comité DESC. 2020a. CESCR Treaty Body Database. General Comments. Acceso 20 enero de 2020. https://tbinternet.ohchr.org/_layouts/15/ treatybodyexternal/TBSearch.aspx? Lang=en\&TreatyID=9\&DocTypelD=11

Comité DESC. 2020b. CESCR Treaty Body Database. Reporting status for Spain. Acceso 30 enero de 2020. https://tbinternet.ohchr.org/_layouts/15/ TreatyBodyExternal/Countries.aspx?CountryCode=ESP\&Lang=EN

Consejo de Derechos Humanos de Naciones Unidas. 2015a. Informe nacional presentado con arreglo al párrafo 5 del anexo de la resolución 16/21 del Consejo de Derechos Humanos. A/HRC/WG.6/21/ESP/1. Oficina de Derechos Humanos del Ministerio de Asuntos Exteriores y de Cooperación de España. Acceso 1 noviembre de 2019. https://undocs.org/es/A/HRC/ WG.6/21/LSO/1 
Consejo de Derechos Humanos de Naciones Unidas. 2015b. Informe de/ Grupo de Trabajo sobre el Examen Periódico Universal. España. A/HRC/29/8. Acceso 1 noviembre de 2019. https://undocs.org/es/A/HRC/29/8/Add.1

Consejo de Derechos Humanos de Naciones Unidas. 2015c. Informe del Grupo de Trabajo sobre el Examen Periódico Universal. España. Adición Observaciones sobre las conclusiones y/o recomendaciones, compromisos voluntarios y respuestas del Estado examinado. A/HRC/29/8/Add. 1. Acceso 1 noviembre de 2019. https://undocs.org/es/A/HRC/29/8/Add.1

Consejo de Derechos Humanos de Naciones Unidas. 2015d. Informe del Grupo de Trabajo sobre el Examen Periódico Universal. España. Matriz de recomendaciones. (UPR of Spain - Second Cycle Thematic list of recommendations). Acceso 1 de noviembre de 2019. https://www.ohchr. org/EN/hrbodies/upr/pages/esindex.aspx

Consejo de Derechos Humanos de Naciones Unidas (CDH) 2020. Examen Periódico Universal - España. Acceso 21 febrero de 2020. https://www. ohchr.org/SP/HRBodies/UPR/Pages/ESIndex.aspx.

Consejo de Europa. 1961. Carta Social Europea. (Entry into Force 26/02/1965). España: BOE núm. 153, de 26/06/1980. Acceso 3 de octubre de 2019: https://www.boe.es/buscar/act.php?id=BOE-A-1980-13567.

Consejo de Europa. 1991. Protocol amending the European Social Charter. Turin 21. X 1991, European Treaty Series No. 142. Acceso 3 de octubre de 2019. https://www.coe.int/en/web/conventions/full-list/-/conventions/ rms/090000168007bd24

Consejo de Europa. 1996. European Social Charter (revised). Strasbourg, Details of Treaty No.163. Entry into Force 01/07/1999. Acceso 3 de octubre de 2019: https://www.coe.int/en/web/conventions/full-list/-/ conventions/rms/090000168007cf93

Consejo de Europa. 2006. New system for the presentation of reports on the application of the European Social Charter. 963 Meeting. Committee of Ministers. Acceso 13 septiembre de 2019. CM (2006) 53

Consejo de Europa. 2008a. Form for the reports to be submitted in pursuance of the 1961 European Social Charter and the 1988 Additional Protocol. Adopted by the Committee of Ministers on 26 March 2008. Acceso 10 septiembre de 2019. https://www.coe.int/en/web/european-social-charter/ drafting-reports

Consejo de Europa. 2008b. Form for the reports to be submitted in pursuance of the European Social Charter (revised). Adopted by the Committee of Ministers on 26 March 2008. Acceso 10 septiembre de 2019. https:// www.coe.int/en/web/european-social-charter/drafting-reports

Consejo de Europa. 2014. Ways of streamlining and improving the reporting and monitoring system of the European Social Charter. 1195 Meeting, 19 March 2014. Committee of Ministers. Acceso 10 septiembre de 2019. CM (2014)26

Consejo de Europa. 2018. Press Briefing elements. Conclusion 2017. European Committee of Social Rights. Acceso 16 diciembre de 2019. https://rm.coe. int/press-briefing-elements-conclusions-2017n/168077fedf 
Consejo de Europa. 2020a. Hudoc Database of the European Social Charter. Acceso 22 enero de 2020. https://hudoc.esc.coe.in

Consejo de Europa. 2020b. Conclusions XXI-4 (2019). Conclusions per country. Spain. Acceso 23 abril de 2020. https://rm.coe.int/rapport-espen/16809cfbea

Faleh Pérez, Carmelo. 2020. «Los dictámenes del Comité de Derechos Económicos, Sociales y Culturales y sus efectos jurídicos en España». En Los efectos jurídicos en España de las decisiones de los órganos internacionales de control en materia de derechos humanos de naturaleza no jurisdiccional, coordinado por C. Fernández de Casadevante, C. (coord.), 65-98. Madrid: Dykinson.

Fernández de Casadevante Romaní, Carlos. 2020. «La obligación del estado de reconocer y aceptar los efectos jurídicos de las decisiones de los órganos internacionales de control en materia de derechos humanos». En Los efectos jurídicos en España de las decisiones de los órganos internacionales de control en materia de derechos humanos de naturaleza no jurisdiccional, coordinado por C. Fernández deCasadevante), 237-278. Madrid: Dykinson.

FOESSA, 2019. VIII Informe FOESSA: La exclusión social se enquista en una sociedad cada vez más desvinculada. Acceso 21 abril de 2020. https:// wWw. foessa.es/viii-informe/

Jimena Quesada, Luis. 2014. «El último bastión en la defensa de los derechos sociales: la Carta Social Europea». Revista Jurídica de la Universidad Autónoma de Madrid, 29: 171-189.

Jimena Quesada, Luis. 2017. «Las grandes líneas jurisprudenciales del Comité Europeo de Derechos Sociales: tributo a Jean-Michel Belorgey». Revista Jurídica de los Derechos Sociales, Lex Social (1): 1-25.

Leppik, Lauri. 2016. "The use of statistical indicators in monitoring social rights under the European Social Charter». 3rd Meeting of the COE-FRA-ENNHRIEQUINET Collaborative Platform on social and economic rights. Belgrado: CoEFRA-ENNHRI-EQUINET Collaborative Platform on Social and Economic Rights.

Macho Carro, Alberto. 2019. "La naturaleza jurídica del Comité de Derechos Económicos, Sociales y Culturales de Naciones Unidas y de sus pronunciamientos. Especial atención al caso español». Papeles el Tiempo de los Derechos 12.

Nolan, Aoife. 2019. Protecting the Child from Poverty: The Role of Rights in the Council of Europe. Council of Europe. Acceso 20 abril de 2020. https:// www.coe.int/en/web/european-social-charter/-/protecting-the-child-frompoverty-the-role-of-rights-in-the-council-of-europe

Oficina de Derechos Humanos. 2018. Informe intermedio de España para el 2. ${ }^{\circ}$ Ciclo del Examen Periódico Universal. Ministerio de Asuntos Exteriores, Unión Europea y Cooperación. Acceso 10 noviembre de 2019. https://lib. ohchr.org/HRBodies/UPR/Documents/Session21/ES/Report2ndCycle.PDF

ONU. 1948. Declaración Universal de Derechos Humanos. Asamblea General. Resolución 217 A (III). Acceso 15 de octubre de 2019. https://www.un.org/ es/universal-declaration-human-rights/. 
ONU. 1966. Pacto Internacional de Derechos Económicos, Sociales y Culturales. Asamblea General. Resolución 2200 A (XXI). Entrada en vigor: 3 de enero de 1976. Acceso 15 de octubre de 2019. https://www.ohchr.org/SP/ Professionallnterest/Pages/CESCR.aspx

ONU. 2020a. "Un experto de la ONU visitará España para investigar los sorprendentes niveles de pobreza». Noticas ONU, Sistema de Alertas, United Nations Global Communications. 23 enero 2020.

ONU. 2020b. «España recibe 275 recomendaciones para mejorar en materia derechos humanos». Noticas ONU, Sistema de Alertas, United Nations Global Communications. 24 enero 2020.

Sanz Caballero, Susana. 2012. "La protección de los derechos socioeconómicos en la Carta Social Europea. Especial referencia a su uso para erradicar la pobreza en Europa». En Estudios de derecho internacional y de derecho europeo en homenaje al profesor Manuel Pérez González (Vol. 1 Tomo 1), editado por Mariano J. Aznar Gómez, 1237-1264. Valencia: Tirant lo Blanch.

Save the Children, 2020. Coronavirus el 60\% de las familias en pobreza ha visto empeorada su situación laboral. Acceso 21 abril de 2020. https:// www.savethechildren.es/actualidad/coronavirus-el-60-de-las-familias-enpobreza-ha-visto-empeorada-su-situacion-laboral

Terrádez Salóm, Daría. 2019. «Lucha contra la exclusión social y la pobreza. La necesaria ratificación de la Carta Social Europea revisada y su protocolo sobre reclamaciones colectivas en España». Revista Jurídica de los Derechos Sociales, Lex Social (9): 379-393.

Van Boven, Theo. 1997. "The international system of human rights: an overview». En Manual on Human Rights Reporting under Six Major International Human Rights Instruments, editado por UN Office of the High Commissioner for Human Rights (OHCHR). HR/PUB/91/1 (Rev.1), 3-16. Ginebra: Naciones Unidas.

Villán Durán, Carlos. 2020. «El valor jurídico de las decisiones de los órganos establecidos en tratados de las Naciones Unidas en materia de derechos humanos». En Los efectos jurídicos en España de las decisiones de los órganos internacionales de control en materia de derechos humanos de naturaleza no jurisdiccional, Fernández de Casadevante, C. (coord.), 99124. Madrid: Dykinson.

Zugasti, Nerea y García, Ángel. 2019. «La recuperación económica se olvida de los más vulnerables. Una lectura de los cambios en el espacio social de la exclusión tras la crisis en España». En VIII Informe FOESSA: La exclusión social se enquista en una sociedad cada vez más desvinculada. Acceso 21 abril de 2020. https://caritasweb.s3.amazonaws.com/mainfiles/uploads/ sites/16/2019/05/3.1.pdf 


\section{Copyright}

Deusto Journal of Human Rights / Revista Deusto de Derechos Humanos is an Open Access journal; which means that it is free for full and immediate access, reading, search, download, distribution, and reuse in any medium only for non-commercial purposes and in accordance with any applicable copyright legislation, without prior permission from the copyright holder (University of Deusto) or the author; provided the original work and publication source are properly cited (Issue number, year, pages and DOI if applicable) and any changes to the original are clearly indicated. Any other use of its content in any medium or format, now known or developed in the future, requires prior written permission of the copyright holder.

\section{Derechos de autoría}

Deusto Journal of Human Rights / Revista Deusto de Derechos Humanos es una revista de Acceso Abierto; lo que significa que es de libre acceso en su integridad inmediatamente después de la publicación de cada número. Se permite su lectura, la búsqueda, descarga, distribución y reutilización en cualquier tipo de soporte sólo para fines no comerciales y según lo previsto por la ley; sin la previa autorización de la Editorial (Universidad de Deusto) o la persona autora, siempre que la obra original sea debidamente citada (número, año, páginas y DOI si procede) y cualquier cambio en el original esté claramente indicado. Cualquier otro uso de su contenido en cualquier medio o formato, ahora conocido o desarrollado en el futuro, requiere el permiso previo por escrito de la persona titular de los derechos de autoría. 